A MATHEMATICAL MODEL

OF THE STRUCTURE AND EVOLUTION

OF SMALL SCALE DISCRETE

AURORAL ARCS

C.E. Seyler

School of Electrical Engineering and

Laboratory of Plasma Studies

Cornell University

Ithaca, N.Y. 14853

LPS 90-1

Jan. 1990 



\title{
A Mathematical Model of the Structure and Evolution of Small Scale Discrete Auroral Arcs
}

\author{
Charles E. Seyler \\ School of Electrical Engineering and Laboratory of Plasma Studies \\ Cornell University, Ithaca, NY 14853
}

\begin{abstract}
A three-dimensional fluid model which includes the dispersive effect of electron inertia is used to study the nonlinear macroscopic plasma dynamics of small scale discrete auroral arcs within the auroral acceleration zone and ionosphere. The motion of the Alfven wave source relative to the magnetospheric and ionospheric plasma forms an oblique Alfven wave which is reflected from the topside ionosphere by the negative density gradient. The superposition of the incident and reflected wave can be described by a steady state analytic solution of the model equations with the appropriate boundary conditions. This two-dimensional discrete auroral arc equilibrium provides a simple explanation of auroral acceleration associated with the parallel electric field. Three-dimensional fully nonlinear numerical simulations indicate that the equilibrium arc configuration evolves three-dimensionally through collisionless tearing and reconnection of the current layer. The interaction of the perturbed flow and the transverse magnetic field produces complex transverse structure that may be the origin of the folds and curls observed to be associated with small scale discrete arcs. Late time transverse electric field power spectra tend towards a universal $\mathbf{k}^{-5 / 3}$ spectral form.
\end{abstract}




\section{INTRODUCTION}

This paper presents a model of the three dimensional evolution of small scale discrete auroral arcs originating from Alfven waves. The model which includes dispersion due to electron inertia, describes the region of the magnetosphere usually referred to as the acceleration zone, and in addition, includes the interaction of the Alfven waves with a collisional inhomogenous ionosphere. Haerendel; 1983, described the concept of oblique Alfven waves and formulated a model of auroral arcs in which he was able to explain some aspects of the transverse electric field structure. In the present paper, it is shown that nonzero electron mass combined with the oblique Alfven wave model leads to a natural explanation of the origin of a steady state parallel electric field as well as a mechanism for three-dimensional instability of the arc.

The most important results emerging from the study of the proposed model may be summarized as follows. The oblique Alfven wave model of auroral arcs [Haerendel, 1983] is extended to include finite electron inertia. Analytic and numerical solutions of this model are found that prove the existence of non-dissipative steady state wave-like solutions which have a complete two dimensional description of the electric and magnetic fields. This description includes a parallel electrostatic field which drives the field aligned current and accelerates the electrons. These east-west aligned, north-south drifting equilibria are consistent with the observed magnetospheric structure of discrete auroral arcs and offer a simple and self-consistent explanation of the origin of parallel electric fields. The two dimensional arc equilibria are threedimensionally unstable to collisionless tearing and reconnection to produce interesting structure in the electric field and current density.

It is very important to understand the physical scale of the phenomena that is being investigated in the present paper. The terminology that exists in the literature is confusing in this regard so an attempt will be made to place previous work in proper perspective to the present. Hallinan and Davis, 1970 refer to a class of discrete auroral phenomena as "small scale auroral arcs" which are also termed "breakup arcs" by Goertz, 1981. These breakup or small scale au- 
roral arcs have a transverse scale of the order of a kilometer and sometimes have such associated features as folds and curls in the terminology of Hallinan and Davis. The spatial scale is related to the collisionless or electromagnetic skin depth due to electron density. Other auroral phenomena which are also termed discrete auroral arcs have a transverse scale (10 to $100 \mathrm{~km}$ ) which in some models depends upon the conductivities of the generator and the ionosphere [Muira and Sato, 1980; Lyons, 1980; Chiu and Cornwall, 1980; Sonnerup, 1980; Lysak, 1985; Lotko et al, 1987]. The relationship between the various types of discrete arcs is not well understood, but it is often observed that the larger type of arcs form first and then break up by forming a system of arcs which are often described as draperies or curtains. A scenario for this breakup was suggested by Goertz, 1981.

The present work is concerned with the former type of discrete arc and the terminology 'discrete auroral arc' adopted in this paper refers to arcs having a transverse scale of the order of the collisionless skin depth. The acceleration zone is defined to be the region where $c^{2} / \omega_{p}{ }^{2} L_{\perp}{ }^{2}$ $\propto \mathrm{B} / \mathrm{n}$ maximizes, where $\mathrm{L}_{\perp}^{2}$ characterizes a geomagnetic flux tube area. For small scale arcs, the acceleration zone is of particular importance since that is where wave dispersion and the parallel electric field maximize.

The two dimensional interaction of Alfven waves with the ionosphere has been considered by several authors [Goertz and Boswell, 1978; Lysak and Carlson, 1981; Lysak and Dum, 1983]. There is much useful information that can be obtained in considering arc evolution in two dimensions, but it is only in three dimensions that nonlinear behavior plays a significant role. In three dimensions, an east-west aligned arc can be unstable to several potential instabilities. Seyler, (1988) in a preliminary study showed that finite electron inertia leads to a rapidly growing collisionless tearing mode of the Alfven wave when it is bounded by a perfectly conducting static ionosphere and has a transverse scale comparable to the electromagnetic skin depth. Seyler found that the ionospheric boundary conditions are very important in determining the nature of the Alfven wave evolution. Specifically, for a boundary condition corresponding to zero parallel current density, an $\mathrm{MHD}$ shear flow instability resulted rather than the tearing 
mode. Given the sensitivity of the evolution upon the ionospheric boundary conditions, a simulation study using a more realistic ionosphere model is needed.

The primary objectives of this paper are to propose a realistic dynamical model to describe the evolution of Alfven waves in the ionosphere-magnetosphere environment and to present numerical simulation results which show the model self-consistently explains the structural morphology associated with discrete arcs and possibly energetic particle acceleration as well.

The paper is organized as follows. Section 2 is concerned with the mathematical model, its derivation and physical properties. The development of the dynamical model proceeds from first principles, relying upon assumptions appropriate for the inner magnetosphere and the ionosphere. In Sec. 3 a stationary two dimensional solution of the equations is presented that represents a simple picture of a small scale non-dissipative discrete arc with the associated transverse electric field, parallel current density and parallel electric field which accelerates the electrons and supports the parallel current. The results of three dimensional simulations are presented in Sec. 4 show that stationary arcs can be unstable to a collisionless tearing mode which may be responsible for the observed transverse structuring in the form of folds and curls. A summary and a discussion of the most important results is given in Sec. 5.

\section{DYNAMICAL MODEL}

\subsection{Derivation}

A dynamical model of the plasma which is valid on spatial scales between say one hundred meters and 100 kilometers transverse to the geomagnetic field and which extends from the E-region ionosphere to several Earth radii into the magnetosphere would be sufficiently complete to contain most features of the interaction of Alfven waves with the ionosphere. Such a model, however, is intractable insofar as one can compute numerical solutions to the complete model without a simplification of the physics and a limitation of the spatial scales involved. In 
this section a model is derived which we believe contains some of the more important aspects of magnetosphere-ionosphere interaction and which is numerically tractable. The basic model consists of the following set of equations, which are a version of the warm quasineutral twofluid equations.

$$
\begin{gathered}
\partial_{t} n+\nabla \cdot(n \mathbf{u})=0 \\
m n\left(\partial_{t} \mathbf{u}+\mathbf{u} \cdot \nabla \mathbf{u}\right)=-\nabla p+\frac{1}{\mu_{o}} \mathbf{J} \times \mathbf{B}-m n v \mathbf{u} \\
\partial_{t} \mathbf{B}=-\nabla \times \mathbf{E} \\
\nabla \times \mathbf{B}=\mu_{o} \mathbf{J} \\
\mathbf{E}+\mathbf{u} \times \mathbf{B}=\mu_{o} \lambda^{2}\left[\partial_{t} \mathbf{J}+\nabla \cdot(\mathbf{u} \mathbf{J}+\mathbf{J} \mathbf{u})\right]+\frac{1}{n e} \mathbf{J} \times \mathbf{B}-\frac{1}{2 n e} \nabla p+\mu_{o} \eta \mathbf{J}
\end{gathered}
$$

where $\mathrm{n}$ is the plasma density, $\mathbf{u}$ is the fluid velocity, $\mathrm{m}$ is the ion mass, $v$ is the ion-neutral collision frequency, $\eta$ is the magnetic diffusivity, $p$ is the total pressure given by $p=n\left(T_{e}+T_{i}\right)$, with $T_{e}=T_{i}=$ constant. The collisionless plasma skin depth is $\lambda=c / \omega_{p}, J$ is the current density, $\mathbf{B}$ is the total magnetic field (geomagnetic plus perturbed) and the electric field $\mathbf{E}$ has both an electrostatic and electromagnetic component and it is determined by the generalized Ohms law, Eq. (5). Equations (1) - (5) are an adequate description of the macroscopic plasma behavior on spatial scales much larger than the ion gyroradius. They do in fact contain considerably more physics than is essential to describe large scale auroral arc behavior in the magnetosphere and upper ionosphere (F-region).

A simplified model may be derived from a systematic expansion in the small parameter $\varepsilon \equiv \omega / \Omega_{\mathrm{i}} \ll 1$. For the region of interest $-1-2 R_{e}$ the relevant nondimensional physical parameters are taken to scale in $\varepsilon$ as follows: 


$$
\begin{array}{llll}
\mathrm{L}_{\perp} / \mathrm{L}_{\|} \sim \varepsilon & \delta \mathrm{B} / \mathrm{B}_{\mathrm{o}} \sim \varepsilon & \delta \mathrm{u} / \mathrm{v}_{\mathrm{A}} \sim \varepsilon & \mathrm{c} / \mathrm{L}_{\perp} \omega_{\mathrm{p}} \sim 1 \\
\delta \mathrm{u} / \mathrm{L}_{\perp} \Omega_{\mathrm{i}}-\varepsilon & \rho_{\mathrm{i}} / \mathrm{L}_{\perp} \sim \varepsilon & \rho_{\mathrm{e}} / \mathrm{L}_{\perp}-\varepsilon^{2} & \mathrm{v}_{\mathrm{e}} / \mathrm{v}_{\mathrm{A}} \sim \varepsilon
\end{array}
$$

For now the collisional transport parameters $v$ and $\eta$ will be taken to be zero which is the case for the magnetosphere. Later they will be restored when the ionosphere is considered.

An important consequence of the ordering assumptions is that the magnetic field may be split up into a geomagnetic component and a perturbed transverse part due to field aligned currents. Accordingly write $\mathbf{B}$ as

$$
\mathbf{B}=\hat{\mathbf{z}} B_{o}+\hat{\mathbf{z}} \times \nabla \psi
$$

where $\psi$ is the perturbed flux function that obeys

$$
\nabla^{2} \psi=\mu_{o} J_{z}
$$

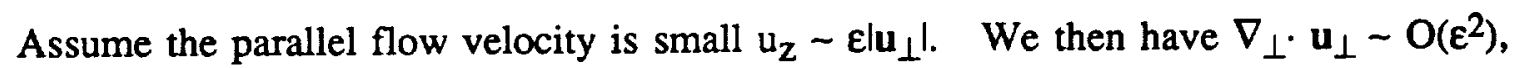
which may taken to be zero. Define the stream function $\phi$ through $\mathbf{u}=\mathbf{z} \times \nabla \phi$. Henceforth let $\nabla \equiv \nabla_{\perp}$ and all $\mathrm{z}$ derivatives will be explicitly displayed as $\partial_{\mathrm{z}}$. To first order in $\varepsilon$ the perpendicular part of the Ohms law becomes,

$$
\mathbf{E}_{\perp}=-B_{o} \nabla \phi
$$

For the $\mathrm{z}$ component the leading order terms are of order $\varepsilon^{2}$,

$$
E_{2}=-\hat{\mathbf{z}} \times \nabla \phi \cdot \nabla \psi+\lambda^{2}\left(\partial_{t}+\hat{\mathbf{z}} \times \nabla \phi \cdot \nabla\right) \nabla^{2} \psi
$$


It will of importance later to consider the parallel electric field given by

$$
E_{\|}=\lambda^{2}\left(\partial_{t}+\hat{\mathbf{z}} \times \nabla \phi \cdot \nabla\right) \nabla^{2} \psi
$$

Taking the $\mathrm{z}$ component of the curl of the momentum equation to leading order in $\varepsilon$, which is order $\varepsilon^{2}$, one obtains assuming the density is constant in $\mathrm{x}$ and $\mathrm{y}$.

$$
\left(\partial_{t}+\hat{\mathbf{z}} \times \nabla \phi \cdot \nabla\right) \nabla^{2} \phi=\frac{1}{m \mu_{o} n_{o}}\left(B_{o} \partial_{z}+\hat{\mathbf{z}} \times \nabla \psi \cdot \nabla\right) \nabla^{2} \psi
$$

To order $\varepsilon^{2}$ the induction equation becomes

$$
\left(\partial_{t}+\hat{\mathbf{z}} \times \nabla \phi \cdot \nabla\right)\left(\psi-\lambda^{2} \nabla^{2} \psi\right)=B_{o} \partial_{z} \phi
$$

Equations (11) and (12) are two equations for the scalar fields $\phi$ and $\psi$. They reduce to the Strauss equations [Strauss, 1976], often called reduced magnetohyrodynamics, for $\lambda^{2}=0$. The collisionless skin depth introduces a length scale into the Strauss equations. Additional physical effects associated with this scale include collisionless tearing and reconnection and a parallel electric field. The discussion on the dynamical invariants of Eqs. (11) and (12) which follows in the next section should provide insight into the role of the collisionless skin depth.

Iit should be pointed out that the ordering assumptions invoked do not include the kinetic Alfven wave discussed by Hasegawa, 1976. This is because for the region of the magnetosphere of interest $<2 R_{e}$, the hybrid ion gyroradius $\rho_{i}=\left(T e^{/ m_{i}} \Omega_{i}^{2}\right)^{1 / 2}$, is considerably less than the skin depth and is therefore not important. The type of Alfven wave that is of interest here is more properly called the inertial Alfven wave. The terminology used in Seyler, 1988 is incorrect in this regard, since that paper dealt with the inertial Alfven wave and not the kinetic Alfven wave of Hasegawa. Although the terminology kinetic has been used to refer to both types of waves, it will not be used here. 


\subsection{Dynamical Invariants}

The magnetospheric plasma evolution equations given by Eqs. (11) and (12) possess two global constant constants of the motion for periodic or perfectly conducting boundary conditions. The total energy $\mathrm{H}$ is conserved and consists of three terms corresponding to transverse kinetic energy, the transverse magnetic energy and the parallel electron kinetic energy due to the current carrying electrons. The form of $\mathrm{H}$ is,

$$
H=\int d^{3} x\left\{|\nabla \phi|^{2}+|\nabla \psi|^{2}+\lambda^{2}\left|\nabla^{2} \psi\right|^{2}\right\}
$$

There is another global invariant which is related to the cross energy of ideal magnetohydrodynamics and is a generalization that includes parallel electron inertia. We denote it by $\Gamma$ and it is,

$$
\Gamma=\int d^{3} x\left\{|\nabla \phi \cdot \nabla \psi|+\lambda^{2} \nabla^{2} \psi \nabla^{2} \phi\right\}
$$

There exists a local invariant which is a generalization of the Alfven frozen flux theorem.

$$
\Phi=\int_{S}\left(\mathbf{B}-\lambda^{2} \nabla^{2} \mathbf{B}\right) \cdot d \mathbf{S}=\int_{c}\left(\psi-\lambda^{2} \nabla^{2} \psi\right) \hat{\mathbf{z}} \cdot d \ell
$$

where $S$ is an open surface and $C$ is the contour bounding that surface. Then generalized flux $\Phi$ is locally conserved, that is it simply advected by the flow and thus obeys,

$$
\left(\partial_{t}+\hat{\mathbf{z}} \times \nabla \phi \cdot \nabla\right) \Phi=0
$$

This invariant shows that the magnetic flux through a surface moving with the fluid is not conserved for $\lambda \neq 0$. Therefore reconnection and tearing of magnetic field lines can occur without resistivity. 


\subsection{Linear Properties}

Consider the dispersion relation for the propagation of a kinetic Alfven wave in a homogeneous equilibrium. We readily find,

$$
\omega^{2}=\frac{k_{z}^{2} v_{A}^{2}}{1+k_{\perp}^{2} \lambda^{2}}
$$

where $\mathrm{k}$ denotes the perpendicular wavenumber. This dispersion relation shows that small transverse scale waves propagate slower than larger scale waves. The consequences of this fact are important for the formation of multiple arc systems. For a spatially localized disturbance the transverse spatial Fourier spectrum of the disturbance is broad. If there is sufficient time for the Fourier components to separate as they propagate along the magnetic field, then the large scale components of the disturbance reach the ionosphere first, followed by short scale components. The effect appears as the breakup of a large scale auroral arc and the formation of a multiple arc system. This scenario for the breakup of arcs was first suggested by Goertz, 1981 .

Now consider the effect of dispersion on the breakup arc after it has formed. The breakup arc has formed an equilibrium that varies in $\mathrm{x}$ and is constant in $\mathrm{y}$. This equilibrium has an $\mathrm{E} \times \mathrm{B}$ flow in the $\mathrm{y}$ direction as well as a perturbed magnetic field also along $\mathrm{y}$, where the ratio of $E_{x} / B_{y}$ is of the order of the Alfven speed $v_{A}$. It is well known that the flow speed must exceed the transverse Alfven speed $\mathrm{v}_{\mathrm{A} \perp}=\mathrm{B}_{\mathrm{y}} / \sqrt{ } 4 \pi \mathrm{m}$ in order that the flow be unstable to the Kelvin-Helmoltz instability , [Chandrasekar, 1961]. So that that the ideal (no dispersion) magnetohydrodynamic $\mathrm{KH}$ instability should not occur in this equilibrium with a large growth rate. However a collisionless tearing mode can be unstable, since dispersion allows for a slippage between the flow and the perturbed magnetic field as is shown by Eq. (15) and Eq. (16). For the case of no equilibrium flow, Seyler, 1988 gave an expression for the linear growth rate of the collisionless tearing mode, which is essentially a re-scaling of the classical result of Furth et al, (1963), and is also given in the review article by White, 1986. The growth rate is, 


$$
\gamma=\mathrm{k} \lambda^{3}\left(\frac{\Delta^{\prime}}{\mathrm{I}}\right)^{2} \tau_{\mathrm{A}}^{-1}
$$

where $\Delta^{\prime}$ is the logarithmic derivative of the outer solution for the perturbed magnetic flux function, $\tau_{\mathrm{A}}$ is the transverse Alfven transit time with respect to the magnetic field produced by field aligned currents and $I$ is a dimensionless integral whose numerical value is about 1 . The important thing to note is the strong dependence of the growth rate upon the skin depth. For typical magnetospheric auroral arc conditions $\mathrm{k} \lambda \approx 1, \Delta^{\prime} \lambda \approx 1$, and $\tau_{\mathrm{A}} \approx 0.2 \mathrm{sec}$, gives $\gamma \approx 5$ $\mathrm{sec}^{-1}$, which is of the order of the observed growth rate for the formation of auroral curls, [Hallinan and Davis, 1970].

\subsection{Magnetospheric boundary conditions}

The magnetospheric boundary corresponds to a generator with an internal conductance G. This boundary condition is identical to that considered by Lysak, 1985 ,

$$
\psi+\mu_{o} B_{o} \Sigma_{G} \phi=f(x, y, t)
$$

where $\Sigma_{G}$ is the generator conductivity and $\mathrm{f}(\mathrm{x}, \mathrm{y}, \mathrm{t})$ is a prescribed function. As Lysak has pointed out this boundary condition corresponds to a pure current generator for $\Sigma_{G} \rightarrow 0$ and a pure voltage generator for $\Sigma_{G} \rightarrow \infty$ and a nonreflecting generator for $\Sigma_{G}^{-1}=\mu_{0} v_{A}\left(1+k^{2} \lambda^{2}\right)^{1 / 2}$.

The form of the function $f$ is taken to be a function of $x$ with small traveling wave perturbations in the $y$-direction and is,

$$
f(x, y, t)=f_{o}(x)\left[1+\sum_{n=1}^{N} C_{n} \sin \left(k_{n} y-\omega t\right) \sin \left(k_{\sigma} x-\omega t\right)\right]
$$

Two forms of $f_{o}(x)$ will be considered. One of which is a Gaussian and the other for 
which $f_{o}^{\prime}(x)$ is a Gaussian. These two forms of $f_{0}(x)$ are intended to model a $V$ shock and an $S$ shock respectively in the terminology of Temerin et al, 1981. Specifically the function used is $f_{0}(x)=\exp \left[-(x-\pi)^{2} / \Delta^{2}\right]$, where $0 \leq x<2 \pi$.

In the simulations to be discussed in Sec. 4 , the magnetospheric boundary condition is chosen to be a non-reflecting generator. For substorm related events, the physical generator is far out in the magnetotail where the slingshot effect launches Alfven waves towards Earth. In the auroral acceleration zone, there is no generator per se, but only the flux of waves from the distant generator. So that by choosing a non-reflecting source of Alfven waves at about $2 R_{e}$ ' the flux of Alfven waves from the actual generator can be modeled without causing non-physical secondary reflections of waves incident on the magnetospheric boundary due to the physical reflections from the ionosphere.

\subsection{Simple Ionospheric Boundary Conditions}

Mallinckrodt and Carlson [1978] considered the reflection of Alfven waves from a conducting ionosphere. For a conducting ionosphere, the reflection coefficient is given by

$$
\Gamma=\frac{R_{i}-Z_{A}}{R_{i}+Z_{A}}
$$

The limiting cases of this expression are: (1) short circuit $\Gamma=-1: \Rightarrow \phi=0$ for $R_{i} \rightarrow 0$,

(2) open circuit $\Gamma=1: \Rightarrow \psi=0$ for $R_{i} \rightarrow \infty$, and (3) nonreflecting $\Gamma=0: \Rightarrow \phi=$ $\mathrm{v}_{\mathrm{A}}\left(1+\mathrm{k}^{2} \lambda^{2}\right)^{1 / 2} \psi / \mathrm{B}_{\mathrm{O}}$

For the naive model of the ionosphere in which $R_{i}=1 / \Sigma_{p}$, a calculation shows for the auroral ionosphere it is generally true that $R_{i} \ll Z_{A}$. In this case $\Gamma \approx-1$, and the ionosphere acts as a short circuit. This assumes, however, that Alfven wave reflections are due to the high conductivity of the ionosphere. As it turns out, a more important source of Alfven wave reflection is due to the negative density gradient and the associated increase in the Alfven speed encountered in the transition from the ionosphere to the magnetosphere, [Ellis and Southwood, 
1983]. This density gradient also gives a reflection coefficient of $\Gamma \approx-1$. The fundamental dif-

ference between the good conductor boundary condition due to small $R_{i}$ and the good reflector boundary condition due to a density gradient is that the good reflector boundary condition alone does not allow a cross field current to close the parallel current. A realistic model of the ionosphere should include a density gradient as well as ion-neutral collisions for current closure.

\section{STEADY STATE AURORAL ARCS}

The model given by Eqs. (11) and (12) admits nontrivial two-dimensional steady state solutions if the the Alfven wave source is drifting normal to the plane of the current sheet and if certain magnetospheric and ionospheric boundary conditions are imposed. The stationary solution to be described is intended to represent a east-west aligned auroral arc during the expansion or recovery phase of an auroral substorm. During the expansion or recovery phase, the auroral oval is expanding or contracting in a north-south direction. This motion is represented by the drift velocity $v_{d}$ of the Alfven wave source. Let the geometry be as follows: $x$ is north, $y$ is east, and $z$ is up. Transform to the frame of the drifting source, then $x \rightarrow x-v_{d} t$. It is important to note that the plasma in the region of interest is not drifting with velocity $v_{d}$, but only the source of Alfven waves. The drifting source of Alfven waves emits what Haerendel [1983] has appropriately called oblique Alfven waves. In steady state, $\partial / \partial t=0$ and in two dimensions $\partial_{\mathrm{y}}=0$, we have,

$$
\begin{gathered}
\mathrm{v}_{d} \partial_{x} \phi+\frac{B_{o}}{m \mu_{o} n_{o}} \partial_{z} \psi=0 \\
\mathrm{v}_{d} \partial_{x}\left(\psi-\lambda^{2} \partial_{x}^{2} \psi\right)+B_{o} \partial_{z} \phi=0
\end{gathered}
$$

These equations may be combined to give 


$$
v_{d}^{2} \partial_{x}^{2}\left(\phi-\lambda^{2} \partial_{x}^{2} \phi\right)=v_{A}^{2} \partial_{z}^{2} \phi
$$

Before presenting the solution to Eq. (24), note that if $\lambda=0$, the form of the equation is a hyperbolic wave equation. Solutions to equations of this type are in general well posed if the boundary conditions are of open surface Cauchy type. For the problem at hand the physically relevant boundary conditions are closed surface Dirichlet, which in general is too restrictive for hyperbolic equations. The general solution of Eq. (24) without application of boundary conditions may be expressed in a Fourier series as

$$
\phi(x, z)=\sum_{k=-\infty}^{\infty} \phi_{k} \exp \left\{i k\left[x \pm \delta\left(1+\lambda^{2} k^{2}\right)^{1 / 2} z\right]\right\}
$$

where $\phi_{k}$ is the Fourier transform of $\phi(x)$ at the magnetospheric boundary. The spatial variables $\mathrm{x}$ and $\mathrm{z}$ are dimensionless and run between 0 and $2 \pi$. The drrift parameter is $\delta=\left(\mathrm{v}_{\mathrm{d}} \mathrm{L}_{\mathrm{z}}\right) /\left(\mathrm{v}_{\mathrm{A}} \mathrm{L}_{\mathrm{x}}\right)$, where $\mathrm{L}_{\mathrm{z}}$ and $\mathrm{L}_{\mathrm{x}}$ are the physical dimensions of the box. The magnetic flux function is,

$$
\psi(x, z)=\mp \sum_{k=-\infty}^{\infty} \frac{\phi_{k}}{\left(1+\lambda^{2} k^{2}\right)^{1 / 2}} \exp \left\{i k\left[x \pm \delta\left(1+\lambda^{2} k^{2}\right)^{1 / 2} z\right]\right\}
$$

The presence of the ionosphere means that the Alfven wave will be reflected. The solution for the case of a perfectly conducting ionosphere $(\phi=0)$ and a nonreflecting generator at the magnetospheric boundary is given by

$$
\psi(x, z)=\sum_{k=-\infty}^{\infty} \frac{f_{k}}{\left(1+\lambda^{2} k^{2}\right)^{1 / 2}} \exp \left\{i k\left[x+2 \pi \delta\left(1+\lambda^{2} k^{2}\right)^{1 / 2}\right]\right\} \cos \left[k z \delta\left(1+\lambda^{2} k^{2}\right)^{1 / 2}\right]
$$

where $f_{\mathrm{k}}$ is the Fourier transform in $\mathrm{x}$ of a time independent arbitrary boundary profile $\mathrm{f}(\mathrm{x})$. 
The steady state parallel electric field, the $\mathrm{x}$-component electric field and the parallel current density can be expressed in terms of the solution, Eq. (27). These are,

$$
E_{\|}(x, z)=-\delta \lambda^{2} \partial_{x}^{3} \psi(x, z)=-\partial_{x} \phi+\delta \partial_{x} \psi
$$

which is found from Eq. (10), the X-component of $E$ is obtained from Eq. (8) and (22),

$$
E_{x}(x, z)=-\delta^{-1} \partial_{z} \psi(x, z)
$$

and the parallel current density is given by Eq. (7)

$$
J_{\|}(x, z)=\partial_{x}^{2} \psi(x, z)
$$

There are several important and interesting features of this solution. Firstly it is obvious that the parallel electric field vanishes when either $\delta=0$ or $\lambda=0$. This means that for a significant parallel electric field to exist it is required that the arc be drifting normal to the direction of its alignment and that the transverse scale be of the order of the skin depth. The physical origin of the parallel electric field may be explained as follows. The quasineutrality condition requires $\partial_{\mathbf{x}} \mathrm{J}_{\mathbf{x}}+\partial_{\mathbf{z}} \mathrm{J}_{\mathbf{z}}=0$, so that the cross field ion polarization current is balanced by the parallel electron current so as to maintain approximate charge balance. If, however, the electrons have finite inertia the parallel current they carry will lag slightly behind the cross field current, producing a small charge imbalance and thus a parallel electric field. Clearly the existence of the parallel electric field is implied by the fact that the region carrying parallel current is drifting into a current free region, hence for finite mass electrons an accelerating parallel electric field is required to maintain a steady state current in the frame moving with $v_{d}$.

Secondly, it is less obvious that $E_{x}$ vanishes if both $\delta=0$ and $\lambda=0$, but it does not vanish if either $\delta$ or $\lambda$ is nonzero. This is physically reasonable, since only if there is no dispersion and no drift will the reflected electric field of the Alfven wave exactly cancel the 
incident field so that the total field is zero.

The steady state solution given by Eqs. (27) - (30) is intended to show the interrelationship of the current and the electric field and it is probably a realistic description for distances far from the ionosphere. An inhomogenous ionosphere, which was not considered in the analytic solution, significantly modifies the field structure near and within the ionosphere. The analytic solution given by Eq. (27) has been verified by comparing it to a two-dimensional time dependent simulation. The results are virtually identical. In the next section the threedimensional time dependent model is extended to include a realistic inhomogeneous ionosphere, and numerical solutions are presented for a variety of situations.

\section{NUMERICAL SiMULATIONS}

\subsection{Inhomogenous Ionosphere Model}

The model equations (11) and (12) are expected to be valid for a homogeneous nondissipative magnetosphere. A realistic ionosphere is both inhomogeneous and collisional; thus to extend the validity of the model into the ionosphere, additional terms must be incorporated to account for the effects of reflection from the density gradient, current closure via Pederson currents and resistive dissipation. To include transport terms, a coordinate transformation along the magnetic field is performed in order to resolve equilibrium ionospheric density variations.

Assume $n_{0}(z)$ depends only upon $z$ and take $B_{0}$ to be constant. Then define a new coordinate along $\mathrm{B}_{\mathrm{o}}$ through $\mathrm{dz} / \mathrm{dq}=\mathrm{v}_{\mathrm{A}}(\mathrm{z})$ for $\mathrm{q}_{1}<\mathrm{q}<\mathrm{q}_{\mathrm{o}}$. Defining $\alpha(\mathrm{q})=\left[\mathrm{n}_{\mathrm{o}}\left(\mathrm{q}_{\mathrm{o}}\right) / \mathrm{n}_{\mathrm{o}}(\mathrm{q})\right]^{1 / 2}$, the final equations are written in the frame moving with the generator are,

$$
\begin{gathered}
{\left[\partial_{t}-v_{d} \partial_{x}+\hat{\mathbf{z}} \times \nabla \phi \cdot \nabla+v(q)\right] \nabla^{2} \phi=\frac{\alpha(q)}{m \mu_{o} n_{o}}\left[B_{o} \partial_{q}+\alpha(q) \hat{\mathbf{z}} \times \nabla \psi \cdot \nabla\right] \nabla^{2} \psi} \\
\left(\partial_{t}-v_{d} \partial_{x}+\hat{\mathbf{z}} \times \nabla \phi \cdot \nabla\right)\left(\psi-\lambda^{2} \nabla^{2} \psi\right)=\frac{B_{o}}{\alpha(q)} \partial_{q} \phi+\eta(q) \nabla^{2} \psi
\end{gathered}
$$


The ion-neutral collision frequency is $v(q)$, and the resistive diffusion coefficient is $\eta(q)$. In this form the equations are suitable for an inhomogeneous ionosphere and they can be stably advanced in time. Without the re-scaling of the coordinate along the magnetic field, structures of a certain size along B will shorten relative to the spatial grid as they propagate into the region where the Alfven phase velocity decreases. This causes numerical instability due to fine scale structure accumulating at the grid scale size.

For the purpose of acheiving Alfven wave reflection and current closure, very simple profiles of $n_{o}(q), v(q)$, and $\eta(q)$ are chosen. All time dependent simulation runs use 48 spatial grid points in the $\mathrm{z}$ direction. Let $\mathrm{j}$ denote the $\mathrm{j}^{\text {th }}$ grid point in $\mathrm{z}$ starting at the bottom of the ionosphere. Then the profile is given by $n_{o j}=\left(256 / j^{2}\right)^{3 / 2}, v_{j}=50(16-j) / 15, \eta_{j}=0.2(16-$ j)/15 for $j=1,16$; and $n_{o j}=1, v_{j}=0, \eta_{j}=0$, for $j=16,48$. While not a completely realistic representation of the ionosphere, this simple profile accomplishes two things. First, Alfven waves are efficiently reflected due to the density gradient, and secondly the profile of the ionneutral collision frequency is adequate to close most of the parallel current. The remaining parallel current is closed by applying the $\phi=0$ boundary condition at the first grid point. It is possible to do a better job of modeling the ionosphere, however, for the purposes of the present paper and to do so would require many more grid points in the $z$ direction which would make the three-dimensional simulations too expensive. In any case it has been found through experimentation with various other ionospheric profiles that the essential results are not changed as long as the criteria of good Alfven wave reflection and good current closure are met.

\subsection{The Numerical Code}

The three dimensional equations are solved using a mixed spectral and finite difference method with a second order Runge-Kutta as the time advance. The nonlinear terms in the transverse plane are computed using fast Fourier transforms and de-aliasing is implemented by grid shifting. The use of second order Runge-Kutta allows one to de-alias without additional 
computational expense. The size of the $x-y$ grid in all runs shown is $32 \times 32$, although some runs were performed using a $64 \times 64$ grid to determine if there were any qualitative differences. There were no significant differences. The $\mathrm{z}$ coordinate is finite differenced with the dependent variables $\phi$ and $\psi$ defined on alternate grid points to avoid instability associated with the decoupling of the grid points. For conserving boundary conditions, the algorithm preserves the two global invariants discussed in section 2 to to better than $0.01 \%$ for 1000 time steps if the parallel and transverse Alfven Courant conditions are satisfied by a factor of two or more.

The equations are solved in an appropriate set of dimensionless variables in which time is normalized to the parallel Alfven transit time. The total length of the system along the geomagnetic field in code units is $2 \pi$. So that one complete transit along B takes $2 \pi$ code time units. There is no fixed value of physical length along $B$, but the ratio of the $x$ and $z$ scale lengths is the same as the ratio of the transverse to parallel Alfven speeds and physically it is of the order of $1 / 1000$. For the purpose of interpreting the simulation data, it is reasonable to take the the $\mathrm{x}$-dimension to be about $10 \mathrm{~km}$, the $\mathrm{y}$ dimension $20 \mathrm{~km}$ and the $\mathrm{z}$ dimension $10,000 \mathrm{~km}$. To convert code time units to seconds, take the average Alfven speed to be $10^{7} \mathrm{~m} / \mathrm{s}$, then $2 \pi$ code time units is one second real time. These numbers should only be considered as guidelines since one is free to choose any set of scales, since the only physical parameter that selects a spatial scale is the ambient magnetosphere plasma density. For example, choosing the $\mathrm{x}$-dimension to be $10 \mathrm{~km}$ and setting $\lambda=1$ in code units, implies the plasma density is about $10 \mathrm{~cm}^{-3}$. Alter-

natively if we take the plasma density to be $1 \mathrm{~cm}^{-3}$ with $\lambda=1$, the the $\mathrm{x}$-dimension of the simulation region is about $33 \mathrm{~km}$.

\subsection{Steady state solutions}

All runs are initialized with zero field within the simulation box. The Alfven wave is launched into the box by application of the non-reflecting magnetospheric boundary condition Eq. (19), which is turned on by ramping the function $\mathrm{f}_{\mathrm{o}}$ linearly in time until $\mathrm{t}=2 \pi$ at which time it is held at a constant amplitude of 2 for the duration of the run. 
Contour plots of the electrostatic potential, the magnetic flux function, parallel electric field, and the parallel current density are presented in Figure 1 and 2 (clockwise starting at the top left panel) for the two-dimensional version of Eqs. (31) and (32) with $\delta=0.1, \lambda=1.0$ for the $\mathrm{S}$ and $\mathrm{V}$ shock cases respectively. All plots are shown in the source drift frame. The results are for time $t=20$, where $t=2 \pi$ is the Alfven transit time along B. For times beyond $t=16$ or so the results do not change significantly and thus a steady state has been achieved.

There are several aspects of two-dimensional arc structure that can be seen from the contour plots. Regarding the closure of potential and parallel current contours, note that most of the potential contours close near the top of the ionospheric region which is consistent with electric field reflections from the density gradient. There are of, course, some potential lines which extend into the closure region (not drawn) to produce the electric field which drives the closure current. Most of the current contours, however, close well within the ionosphere, since the closure Pederson currents require ion-neutral collisions and higher plasma density.

A comparison of the structure of the parallel current density and the parallel electric electric field shows that the positive parallel electric field occurs in the region of negative $\partial_{x} J_{\|}$. Thus the rightward drifting positive parallel electric field accelerates electrons downward to produce the upward current which lags behind the parallel electric field.

\subsection{Three-dimensional time dependent simulations}

The two-dimensional steady state solutions do not remain two-dimensional if they are perturbed in the $y$ coordinate. The results of three-dimensional nonlinear simulations are presented for the same V shock and S shock magnetospheric boundary conditions as the two-dimensional runs except that a small symmetry breaking perturbation is applied in the form given by Eq. (20). In order to see the effect of the source drift on the three-dimensional evolution, the drift parameter $\delta$ is taken to be 0.0 and 0.1 for each of the $V$ and $S$ shock cases. A total of four runs are presented with the simulation parameters parameters given in table 1.

In Figures 3-6 are the main results from the three-dimensional simulations correspond- 
ing to runs 1 through 4 respectively. The differences between the non-drifting $(\delta=0)$ and drifting $(\delta=0.1)$ runs are discussed first. The main qualitative difference is the asymmetry about the $x=\pi$ plane which is also present in the two-dimensional simulations. However, in the three-dimensional simulations for the $S$ shock case, the asymmetry is also reflected in the qualitative differences between the negative and positive values of the potential and the current density in the $x-y$ plane. For the $V$ shock case, the main difference between the $\delta=0$ run and the $\delta=0.1$ run is that the non-drifting case has somewhat more structure than the drifting case. It was observed in other runs as well (not shown) that increasing $\delta$ decreased the growth rate of the instability for both $\mathrm{S}$ and $\mathrm{V}$ shock boundary conditions.

The structure along the magnetic field is much more field aligned than one would be led to believe from the $\mathrm{x}-\mathrm{z}$ contour plots in the lower panels of Figures 3 - 6. This is because the total magnetic field, geomagnetic plus that due to $\mathrm{J}_{\|}$, has field lines that make a large angle with respect to the vertical in the $y-z$ plane due to the strongly contracted $z$-dimension. Thus structure existing in the $x-y$ plane is projected onto the $x-z$ plane. This is clearly seen by comparing the current density plot in the $x-y$ and the $x-z$ planes. Note also that there is much structure in the parallel electric field. The reason for this is the parallel electric field has one more derivative and hence more structure than the current density (see Eq. (28)).

The source of the instability which is the origin of the three-dimensional structuring in all runs is the lowering of the magnetic energy through magnetic reconnection, i.e. a tearing mode. A tearing mode is implied for two reasons. One is because the local magnetic energy density exceeds the local flow kinetic energy and the form of the magnetic flux function and the flow stream function are similar. If the reverse were true then a shear flow instability like KelvinHelmholtz would be more likely. A stronger indication is that the hallmark of a tearing mode is found in the form of the current density viewed in the $x-y$ plane. This is the formation of current filaments at magnetic $x$-points and flow vortices acting such as to force fluid into the magnetic islands between the filaments. The collisionless tearing instability as a possible source of auroral structuring was first suggested by Seyler, 1988. 
In Figure 7 is a time sequence of contour plots of the electrostatic potential. The upper left plot is at time 16, the upper right at time 17, the lower left at time 18 and the lower right is at time 19. The entire sequence occurs on a real time scale of the order of a second. This is of the same order as that observed by Hallinan and Davis, 1970, and it is consistent with the estimated value of the tearing mode growth rate given in Sec. 2.3.

The spectral characteristics of the instability in the nonlinear turbulent regime are quite interesting. The one dimensional power spectra of the potential (stream function) are presented in Figures $8-11$. The first panel in each Figure is the $\mathrm{x}$-direction power spectrum which is a function of $k_{x}$ and an average over $k_{y}$. The second panel are the corresponding $y$-direction power spectra. Figures 8 and 9 , which correspond to the $S$ shock cases, are instantaneous power spectra at time 20, while Figures 10 and 11 corresponding to the $V$ shock case are taken at time 18. A qualitative description of the spectral behavior of the electric field spectral index, which is 2 lower than the potential spectral index, may be stated as follows. During early stages of the instability, the spectra are much steeper (spectral index -3 to -5 ) than at later times when the flow can be considered to be more turbulent. At late times the spectral index very closely approaches $-5 / 3$, i.e. Kolmogorov scaling. The spectral data presented in Figure $8-11$, correspond to the same times as in Figures $3-6$. A comparison of the results of the $S$ shock cases to the $\mathrm{V}$ shock cases shows that the spectrum is considerably steeper for the $\mathrm{S}$ shock cases. One must be careful in interpreting this observation, because spectra taken at later times for the $\mathrm{S}$ shock case (not shown) also shows a tendency towards a $-5 / 3$ power law. The correct interpretation of this result is that all cases approach a universal power law of $-5 / 3$, and the $S$ shock cases shown represent spectra taken in an earlier stage of evolution. The $S$ shock case is more slowly evolving because the amplitude of the magnetospheric boundary condition is such that the shear in the flow and the strength of the parallel current density is less than the $V$ shock case, compare the values of the current density labels in Figures 1 and 2.

The magnetic field spectrum has also been examined. It appears from the numerical results that the linear relation Eq. (26) holds between the electrostatic potential and the magnetic 
flux function; so that the electric amd magnetic field spectra are related as

$$
\left\langle\left|\mathbf{B}_{k}\right|^{2}\right\rangle=\frac{\left\langle\left.\mathbf{E}_{k}\right|^{2}\right\rangle}{1+\lambda^{2} k^{2}}
$$

\section{SUMMARY AND DISCUSSION}

\subsection{Summary}

The primary conclusions that can be drawn from the analytical and numerical studies of the model are as follows:

1. Oblique Alfven waves can form two dimensional steady state auroral arcs. For a nonzero collisionless skin depth there is associated a parallel electric field that drives the parallel current of the oblique Alfven wave.

2. Density gradients as found in the topside ionosphere act as a good reflector of Alfven waves with an electric field reflection coefficient of $\Gamma=-1$. If the generator source is drifting relative to the plasma (oblique Alfven wave) then ionospheric reflections do not completely cancel and lead to a net transverse electric field.

3. Two dimensional steady state arcs are unstable to three-dimensional perturbations. The arcs evolve through a combination of collisionless tearing and shear flow, with stronger driving field aligned current resulting in more rapid three-dimensional evolution.

4. The transverse electric field spectrum of a three-dimensionally evolving arc appears to approach a $\mathrm{k}^{-5 / 3}$ power law as time progresses. The approach is faster for higher shear and parallel current. At earlier times the spectrum may be quite steep.

The results of the equilibrium model taken together with the results of the simulations indicate that the ionosphere play at least two principal roles in magnetosphere-ionosphere coupling. The first, and perhaps under appreciated, is the density gradients at high altitudes that 
demark the upper boundary of the ionosphere $(350-1000 \mathrm{~km})$ can reflect Alfven waves quite efficiently. The combined effect of the incident and reflected oblique Alfven waves together with their response to electron inertia produces the electric field and parallel current structure of auroral arcs. The second (traditional) role is E-region current closure which closes the current carried by the electron beam that constitutes most of the upward parallel current. The electron current penetrates to much lower altitude than where Alfven waves are reflected and therefore has approximately zero parallel gradient, and hence no associated cross field closure currents. At such altitudes ( $110-160 \mathrm{~km}$ ) where the ion-neutral collision frequency is sufficiently large to allow ion cross field currents is the circuit completed.

\subsection{Discussion}

The stationary arc solutions discussed in Secs. 3 and 4.3 clearly demonstrate the existence of the parallel electric field, an estimate of the magnitude of $E_{\|}$may be obtained from Eqs. (10) or (28).

$$
E_{\|} \approx \frac{m_{e} v_{d}}{n_{o} e^{2}} \partial_{x} J_{\|}
$$

The quantities appearing in Eq. (34) are estimated as follows. $\partial_{\mathrm{x}} \sim 2 \times 10^{-3} \mathrm{~m}^{-1}, \mathrm{n}_{\mathrm{o}} \sim 10^{6} \mathrm{~m}^{-3}$, $\mathrm{J}_{\|} \sim 10^{-6} \mathrm{~A} / \mathrm{m}^{2}$ and guess the drift velocity to be $\mathrm{v}_{\mathrm{d}} \sim 3,000 \mathrm{~m} / \mathrm{s}$ in the magnetosphere which maps to a much lower velocity in the ionosphere. With these values, $E_{\|} \sim 0.2 \mathrm{mV} / \mathrm{m}$, which is equivalent to one $\mathrm{keV}$ per $5000 \mathrm{~km}$ along $\mathrm{B}$. The esimates of the parameters are typical, but any one may vary by a factor of two or more. It would seem to be possible to achieve a parallel electric field of $1 \mathrm{mV} / \mathrm{m}$ and thereby accelerate electrons to a maximum energy of about $20 \mathrm{keV}$ without requiring unrealistic magnetospheric conditions.

Some comments are in order regarding observed electron precipitation data and possible connections with the model discussed here. The sounding rocket data of Arnoldy, 1970, showed rapid temporal variations of electron current and energy. This may have been be due to 
internal reflections of the Alfven waves due to the magnetospheric density structure, Lysak, 1988. It is possible to simulate auroral flicker using the model in this paper, but it would require a considerably more detailed magnetospheric density and magnetic field profile than was used.

Recent data high resolution sounding rocket data, given in Earle [1988] and provided by C. Kletzing and R. Torbert [unpublished], clearly shows the breakup arcs as inverted V's of about $1 \mathrm{keV}$ energy and a width of less than one kilometer. The electron precipitation satellite data of Gurnett and Frank, 1973, is not of sufficient resolution to detect the structure of the breakup arcs, as is the case for all satellite data that the author is aware.

Whalen and Daly, 1979, have argued that static parallel electric fields will not produce the flat parallel electron distributions that they observed. In the present model the parallel electric field is not static relative to the background plasma, but rather it drifts into a region of stationary electrons. So that electrons originating from different altitudes along the same magnetic field line will acquire different energies by the time they reach the observation point below. Therefore, it does not seem that the data of Whalen and Daly are inconsistent with the model proposed here for electron acceleration. This point cannot be argued much further without a two-dimensional test particle simulation of electrons in the fields produced by a stationary arc. This topic is currently being investigated.

The structuring found in the three-dimensional simulations bears resemblance to the observations of Hallinan and Davis, 1970; however is difficult to assess just what simulation data should be compared to the observations of auroral curls. The potential contours bear the closest resemblance to the curls, but one might expect that the current density would be more appropriate for comparison. This is a rather difficult issue to resolve, since this kind of qualitative comparison is highly subjective. Nevertheless it is fair to say that the collisionless tearing mode does have a structural morphology similar to auroral curls, as does the Kelvin-Helmholtz instability, Keskinen et al, 1988, although the author does not believe that a shear flow instability is the origin of auroral curls in small scale arcs. The Kelvin-Helmholtz instability probably does play a role in large scale structuring associated with the low latitude boundary layer [Lotko and 
Schultz, 1988] and the magnetopause[Miura, 1984; Wu, 1986].

The spectral features of the electric field produced by the tearing mode process in the nonlinear regime appear to be universal taking on the form of a $-5 / 3$ power law. The DE 2 data of Basu et al, 1988 upon a first consideration seems to be in only partial agreement with the simulations. Three is, however, an interpretation that reconciles the apparent differences between the simulations and the DE 2 data as interpretated Basu et al. First a summary of the observational results as characterized by Basu et al. They found for arcs having strong shear, the spectral index of the electric field was approximately -1.7 , which agrees with the simulations; while for arcs having what was characterized as moderate shear, the spectrum was steeper in the range -2.5 to -3.5 . A reconciliation of the differences with the moderate shear cases, is as follows. From the simulations it was found that lower shear and parallel current resulted in slower evolution of the instability and steeper spectra at the same absolute time (recall the $\mathrm{S}$ shock spectral data). Therefore it is likely that the moderate shear spectra of Basu et al represents the instability in an early stage of evolution when the spectrum is steeper, while the strong shear arcs rapidly evolve towards the universal $-5 / 3$ spectral slope. Basu et al, 1988 also present density power spectra, which we cannot comment upon, since the present model does not have density evolution. This is a subject for future research.

The macroscopic fluid model described here has obvious limitations. Perhaps the most important of these is the simplified description of the electrons in which kinetic effects are modeled through the parameter $\lambda$. Ions as well are treated as a low frequency fluid, so the physics of ion cyclotron waves is completely suppressed. Weak double layers (WDL's) require a full kinetic description of both species are are not described by the present model. It has been conjectured that WDL's may be responsible for auroral electron acceleration, and there is some circumstantial evidence that the cumulative parallel potential drop of many WDL's may suffice to accelerate electrons to $\mathrm{keV}$ energy [Hudson et al, 1983; Koskinen et al, 1988; Tetreault, 1988]. It is, however, the author's belief that WDL's are the result of electron drift and not the origin of the drift. The instability which forms the WDL's (ion-acoustic or possibly something 
else) may act to redistribute the field aligned potential drop into discrete packets, whereas the actual origin of the parallel electric field is due to the oblique inertial Alfven wave.

\section{Acknowledgements}

The author has benefited greatly from discussions with Prof. R. L. Lysak, Dr. W. B. Lotko, Prof. N. F. Otani, Prof. M. C. Kelley and Prof. P. M. Kintner. This work was supported through NSF Grant ATM-8900357 and NASA Grant NAGW-1581. 


\section{REFERENCES}

Arnoldy, R. L., Rapid fluctuations of energetic auroral particles, J. Geophys. Res. 75, 228, 1970. Basu, Sunanda., Santimay, Basu, E. MacKenzie, P. F. Fougere, W. R. Coley, N. C. Maynard, J. D. Winningham, M. Sugiura, W. B. Hanson, and W. R. Hoegy, Simultaneous density and electric field fluctuation spectra associated with velocity shears in the auroral oval, $J$. Geophys. Res., 93, 115, 1988.

Chandrasekar, S., Hydrodynamic and Hydromagnetic Stability, Clarendon press (Oxford), Chapter XI, 1961.

Chiu, Y. T. and J. M. Cornwall, Electrostatic model of a quiet auroral arc, J. Geophys. Res., 83, $543,1980$.

Earle, G, Electrostatic plasma waves and turbulence near auroral arcs, Ph.D. Thesis, Cornell University, 1988.

Ellis, P. and D. J. Southwood, Reflection of Alfven waves by nonuniform ionospheres, Planet. Space Sci., 31, 107, 1983.

Furth, H. P., J. Killeen, and M. N. Rosenbluth, Finite resistivity instabilities of a sheet pinch, Phys. Fluids, 6, 459, 1963.

Goertz, C. K., and R. W. Boswell, Magnetosphere-ionosphere coupling, J. Geophys. Res., 84, $7239,1979$.

Goertz, C. K., Discrete Breakup Arcs and Kinetic Alfven Waves, in Physics of Auroral Arc Formation, ed. by S.I. Akasofu and J.R. Kan, AGU Monograph 25, 451, 1981.

Gurnett, D. A. and L. A. Frank, Observed releationships between electric fields and auroral par ticle precipitation, J. Geophys. Res., 78, 145, 1973.

Hasegawa, A., Particle accleleration by MHD surface wave and formation of the aurora, $J$. Geophys. Res., 81, 5083, 1976.

Haerendel, G., An Alfven Wave model of Auroral Arcs, in High Latitude Space Plasma Physics, Hultqvist and T. Hagfors, 1983.

Hallinan, T. J. and T. N. Davis, Small scale auroral arc distortions, Planet. Space Sci., 18, 1735, 1970.

Hudson, M. K., W. Lotko, I. Roth, and E. Witt, Solitary waves and double layers on auroral field lines, J. Geophys. Res., 88, 916, 1983.

Keskinen, M. J., H. G. Mitchell, J. A. Fedder, P. Satyanarayana, S. T. Zalesak and J. D. Huba, Nonlinear evolution of the Kelvin-Helmholtz instability in the high latitude ionosphere, J. Geophys. Res., 93, 137, 1988.

Koskinen, H. R., R. Bostrom, and B. Holback, Viking observations of solitary waves and weak double layers on auroral field lines in Ionosphere-Magnetosphere-Solar Wind Coupling Processes, T. Chang, G. B. Crew, and J. R. Jasperse (eds.), Scientific, Cambridge, MA, 1988.

Lotko, W. B., B. Sonnerup, and R. L. Lysak, Nonsteady boundary layer flow including ionospheric darg and parallel electric fields, J. Geophys. Res., 92, 8635, 1987. 
Lotko, W. and C. G. Schultz, Internal shear layers in auroral dynamics, in Modeling Magnetospheric Plasma, T.E. Moore and J. H. Waite (eds.) AGU Monograph 44, American Geophysical Union, Washington, p 121, 1988.

Lysak, R. L. and C. W. Carlson, Effect of microscopic turbulence on magnetosphere-ionosphere coupling, Geophys. Res. Lett., 8, 365, 1983.

Lysak, R. L. and C. T. Dum, Dynamics of magnetosphere-ionosphere coupling including turbulent transport, J. Geophys. Res., 88, 365, 1983.

Lysak, R. L., Auroral Electrodynamics with Current and Voltage Generators, J. Geophys. Res., $90,4178,1985$.

Lysak, R. L., Theory of auroral zone PiB pulsation spectra, J. Geophys. Res., 93, 5942, 1988.

Lyons, L. R., Generation of large-scale regions of auroral currents, electric potentials and precipitation by the divergernce of the convection electric field, J. Geophys. Res., 85, 17, 1980.

Mallinckrodt, A. J. and C. W. Carlson, Relations between transverse electric fields and fieldaligned currents, I. Geophys. Res., 83, 1426, 1978.

Muira, A. and T. Sato, Numerical simulation of the global formation of auroral arcs, J. Geo phys. Res., 85, 73, 1980.

Muira, A., Anomalous transport by magnetohydrodynamical Kelvin-Helmholtz instabilities in the solar wind-magnetosohere interaction, J. Geophys. Res., 89, 801, 1984.

Seyler, C. E., Nonlinear 3-D evolution of bounded kinetic Alfven waves due to shear flow and collisionless tearing instability, Geophys. Res. Lett., 15, 756, 1988.

Sonnerup, B. U. O., Theory of the low latitude boundary layer, J. Geophys. Res., 85, 2017, 1980.

Strauss, H.R., Nonlinear Three-dimensional Magnetohydrodynamics of Noncircular Tokamaks, Phys. Fluids, 19, 134, 1976.

Temerin, M., M. H. Boehm, and F. S. Mozer, Paired electrostatic schocks, Geophys. Res. Lett., 8, 799, 1981.

Tetreault, D. J., Growing ion holes as the cause of auroral double layers, Geophysical Res. Lett., 15, 164, 1988.

Vickrey, J. F., R. C. Livingston, N. B. Walker, T. A. Potemra, R. A. Heelis, M. C. Kelley, and F. J. Rich, On the current-voltage relationship of the magnetospheric generator at intermediate spatial scales, Geophys. Res. Lett., Vol. 13, No. 6, 495, 1986.

Whalen, B. A. and P. W. Daly, Do field-aligned auroral particle distributions imply acceleration by quasistatic parallel electric fields?, J. Geophys. Res., 84, 4175, 1979.

White, R. B., Resistive reconnection, Rev. Mod. Phys., 58, No. 1, 183, 1986.

Wu, C. C., Kelvin-Helmholtz instability at the magnetopause boundary, J. Geophys. Res., 91, $3042,1986$. 
TABLE 1. Parameters for three-dimensional simulations

\begin{tabular}{|c|c|c|c|c|c|c|c|c|c|c|}
\hline Run \# & 1 & $S$ or V shock & 1 & $\delta$ & 1 & $\Delta$ & ! & $\lambda$ & 1 & $\Delta \mathrm{t}$ \\
\hline 1 & i & $S$ & $i$ & 0.0 & $i$ & 17.8 & i & 1.0 & i & 0.005 \\
\hline 2 & 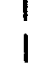 & $S$ & 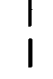 & 0.1 & 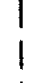 & 17.8 & i & 1.0 & 1 & 0.005 \\
\hline 3 & 1 & V & 1 & 0.0 & 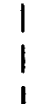 & 17.8 & 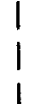 & 1.0 & 1 & 0.005 \\
\hline 4 & 1 & V & $i$ & 0.1 & $i$ & 17.8 & $i$ & 1.0 & $i$ & 0.005 \\
\hline
\end{tabular}



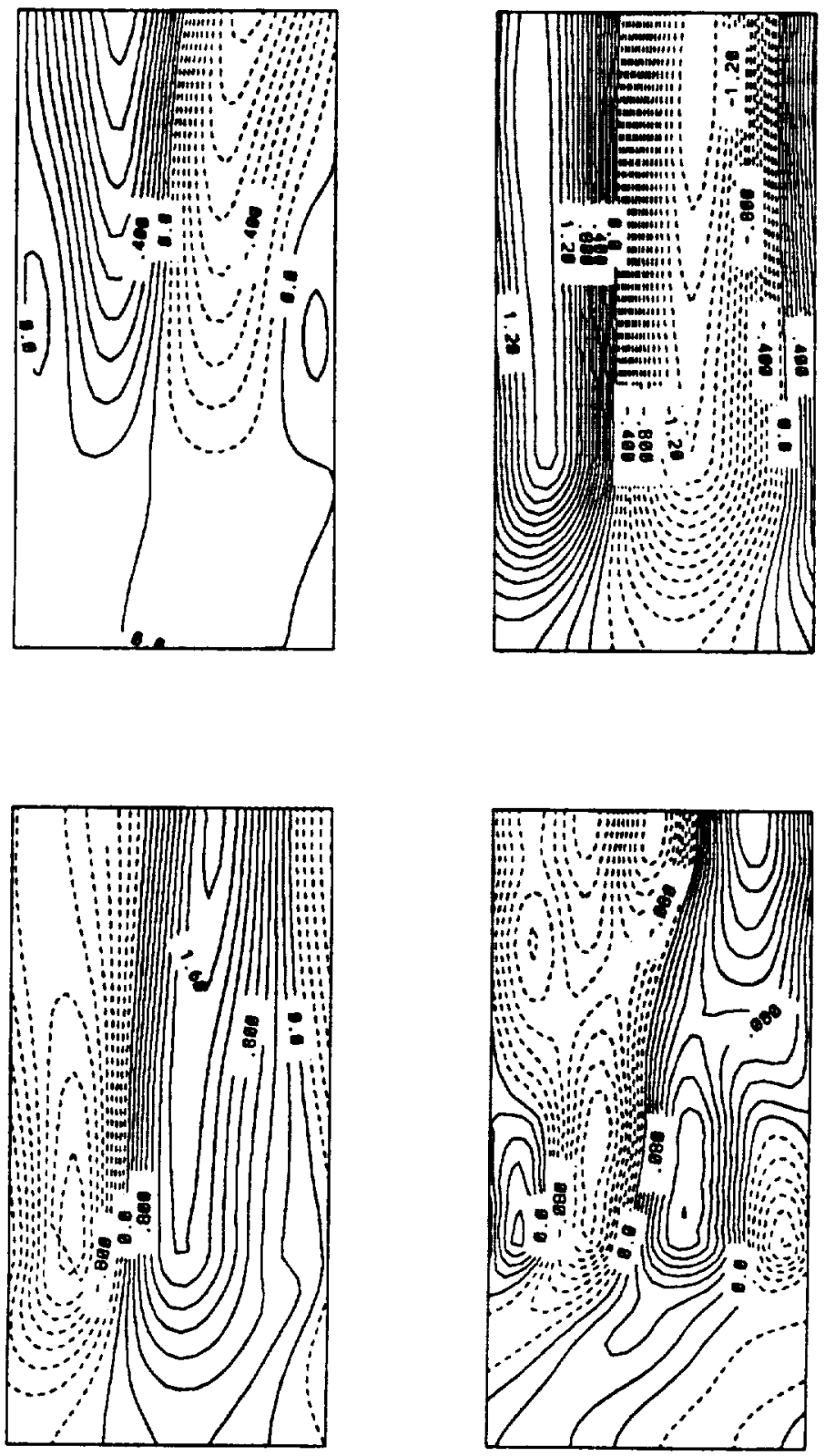

Fig. 1 Contour plots in the $x-z$ plane of the electrostatic potential, magnetic flux function, parallel electric field and parallel current density for the S shock steady state arc, starting at upper right and going clockwise. 

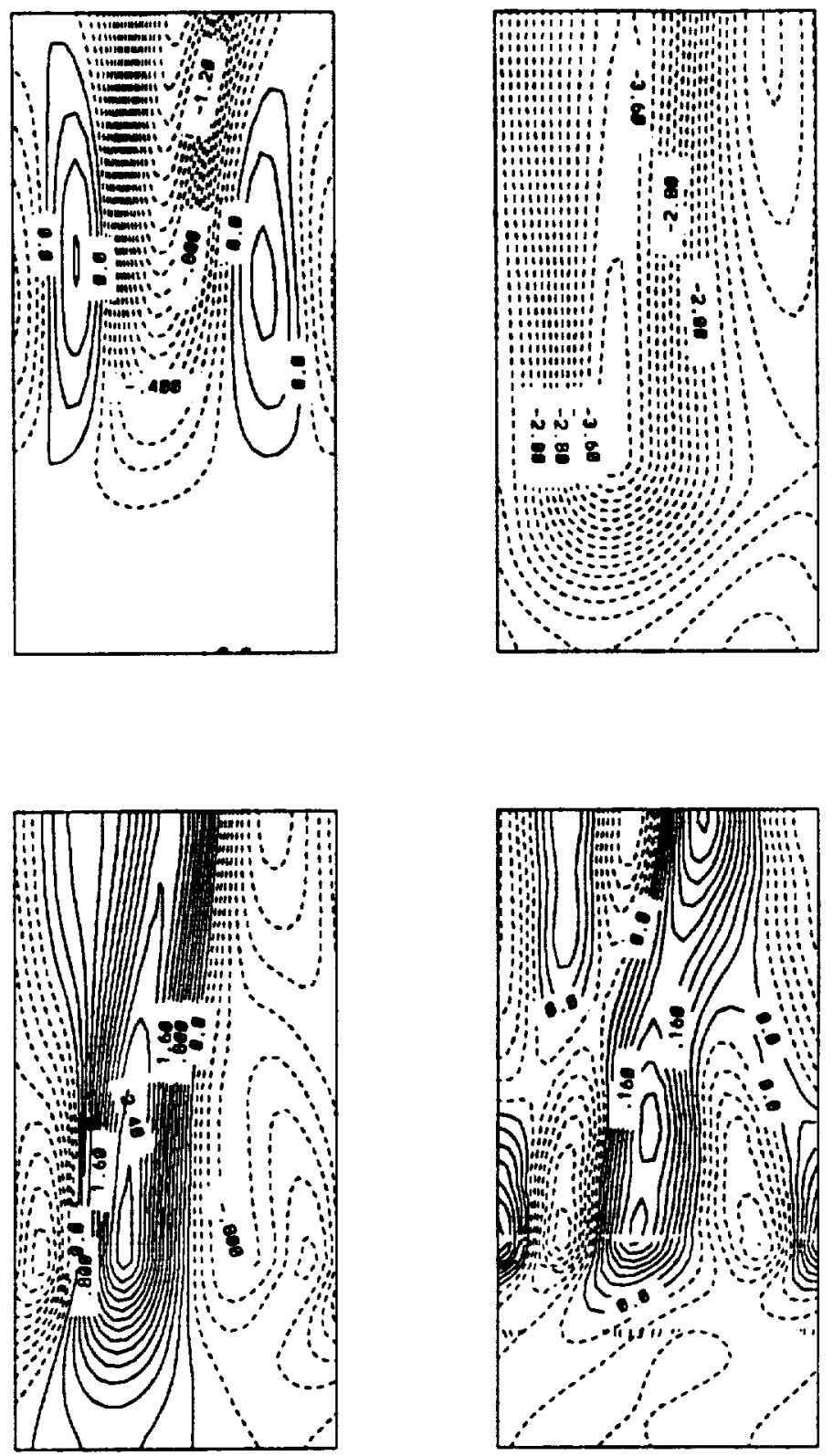

Fig. 2 Contour plots in the $\mathrm{x}-\mathrm{z}$ plane of the electrostatic potential, magnetic flux function, parallel electric field and parallel current density for the $\mathrm{V}$ shock steady state arc, starting at upper right and going clockwise. 

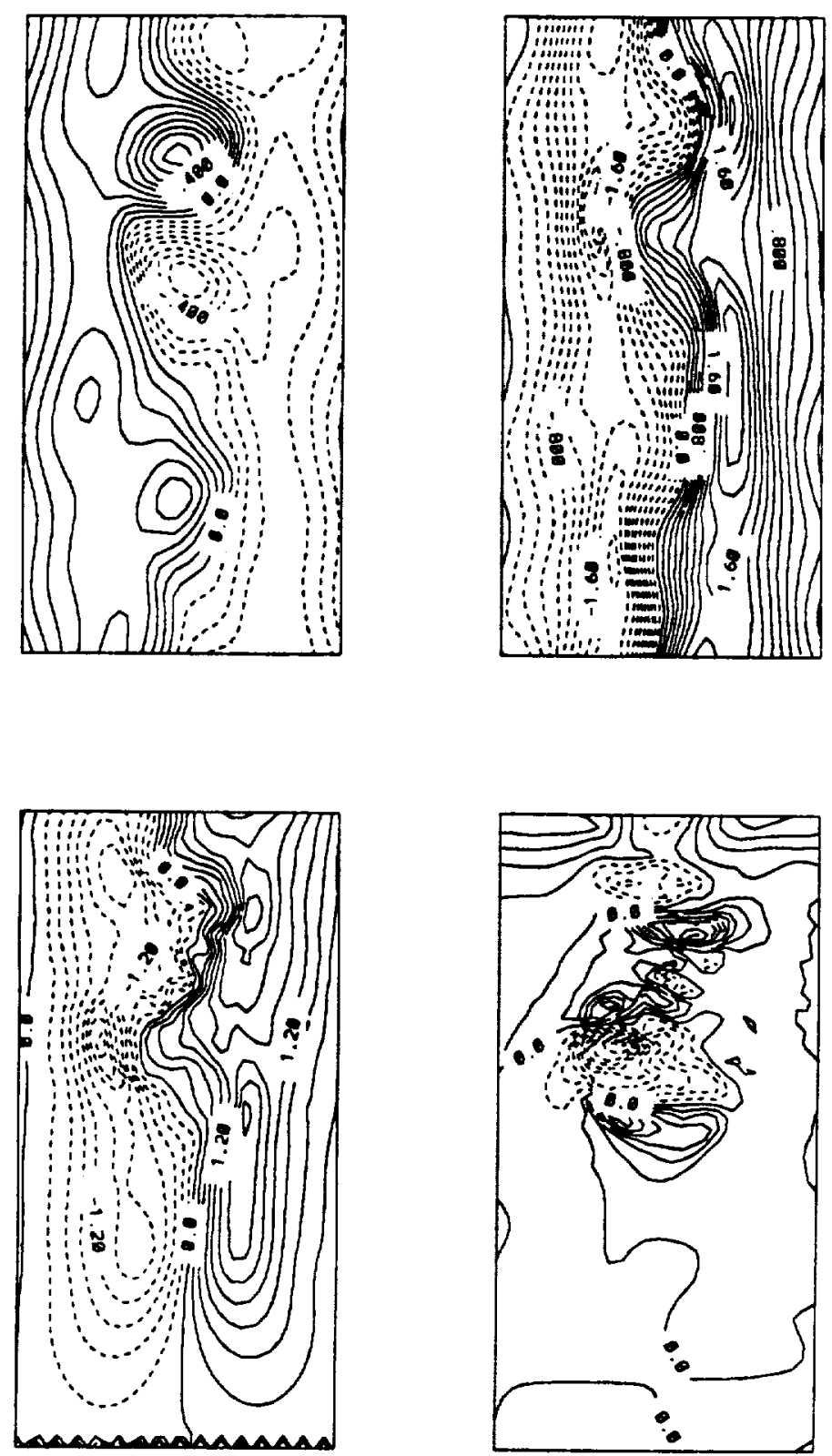

Fig. 3 Contour plots of the electrostatic potential in the $z=\pi$ plane, parallel current density in the $z=\pi$ plane, parallel electric field in the $y=\pi$ plane and parallel current density in the $y=\pi$ plane for run 1 starting at upper right and going clockwise. 

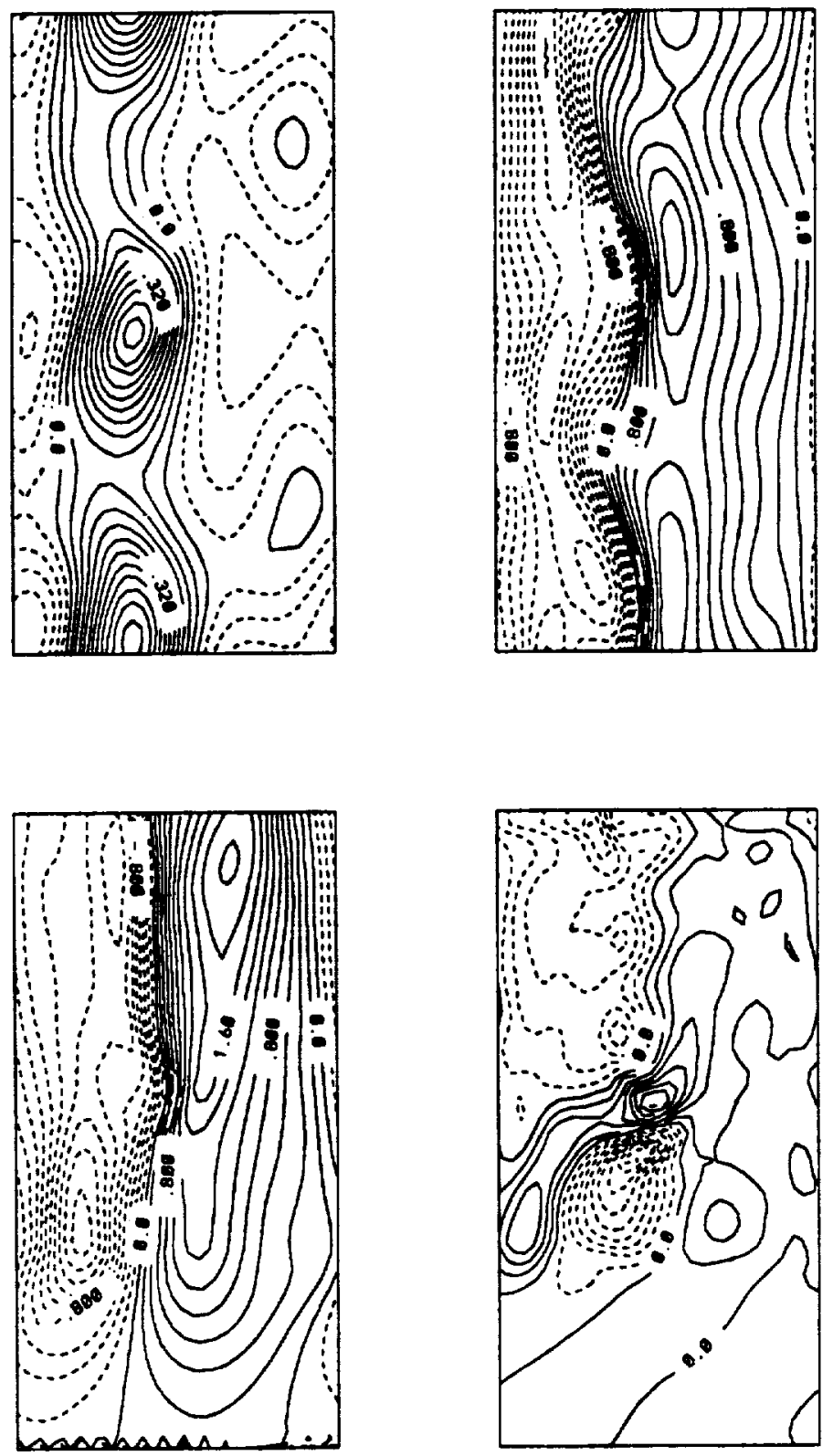

Fig. 4 Contour plots of the electrostatic potential in the $z=\pi$ plane, parallel current density in the $z=\pi$ plane, parallel electric field in the $y=\pi$ plane and parallel current density in the $y=\pi$ plane for run 2 starting at upper right and going clockwise. 

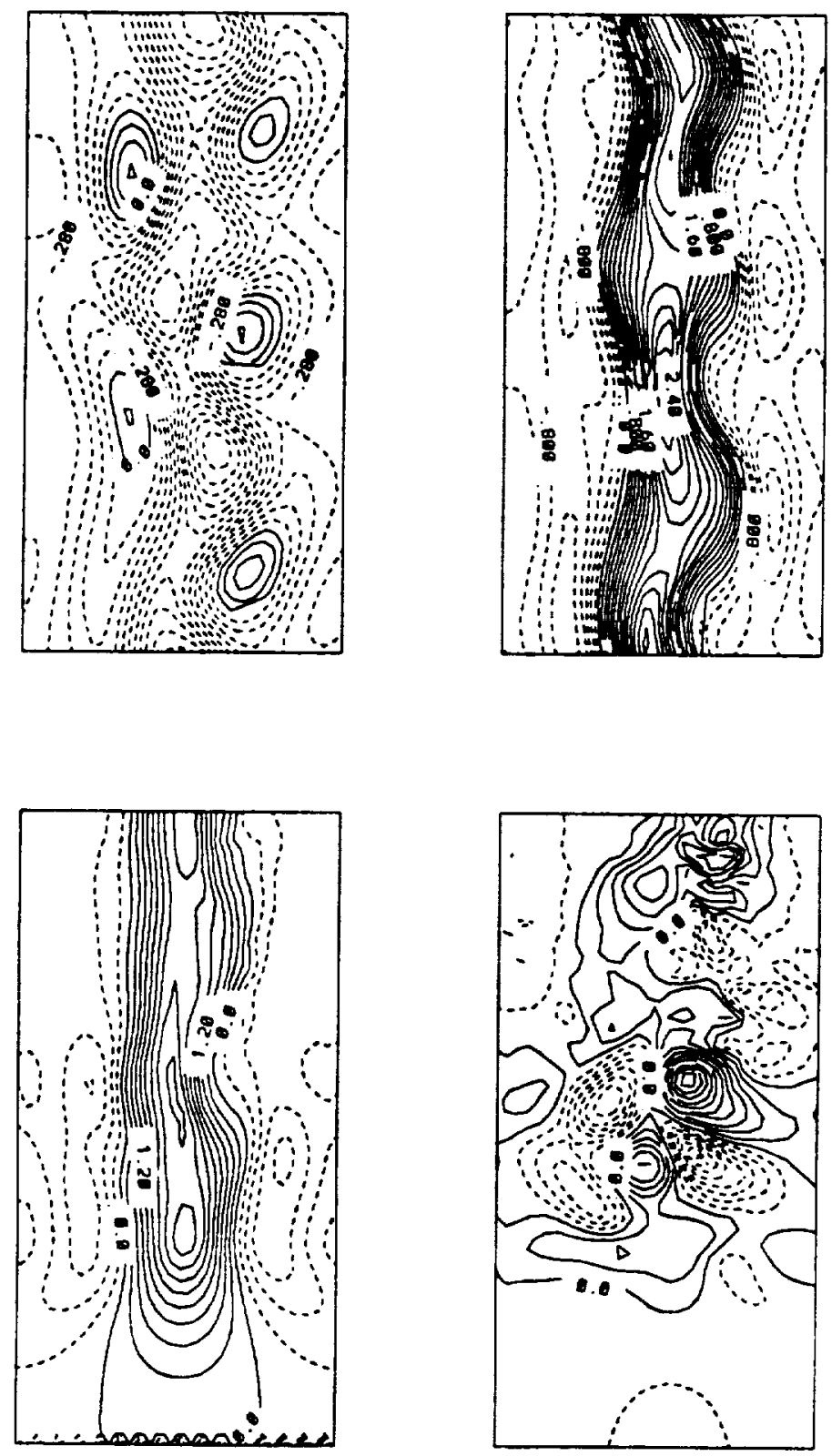

Fig. 5 Contour plots of the electrostatic potential in the $z=\pi$ plane, parallel current density in the $z=\pi$ plane, parallel electric field in the $y=\pi$ plane and parallel current density in the $y=\pi$ plane for run 3 starting at upper right and going clockwise. 

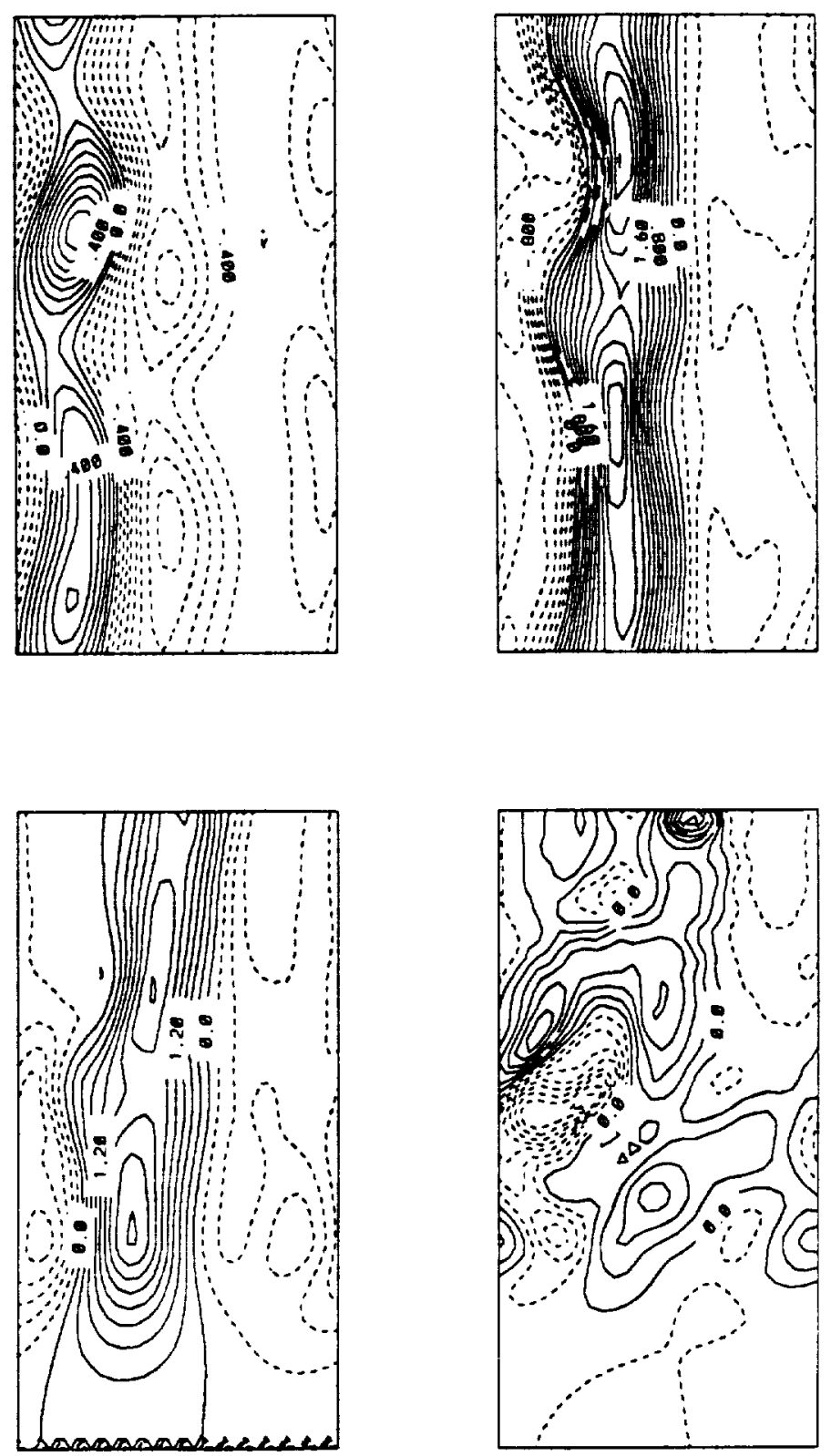

Fig. 6 Contour plots of the electrostatic potential in the $z=\pi$ plane, parallel current density in the $z=\pi$ plane, parallel electric field in the $y=\pi$ plane and parallel current density in the $y=\pi$ plane for run 4 starting at upper right and going clockwise. 

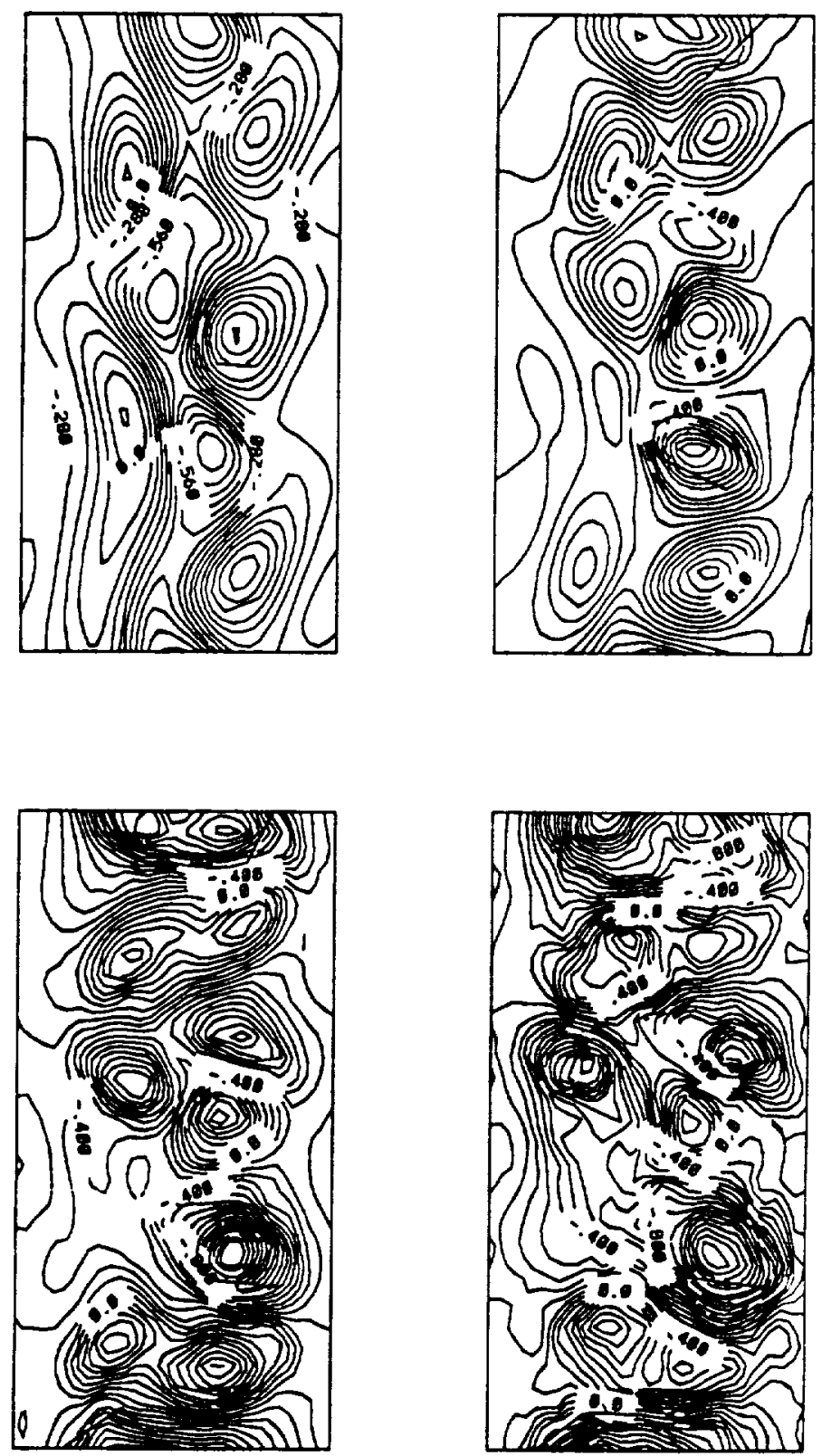

Fig. 7 Time sequence of contour plots of the electrostatic potential in the $y=\pi$ plane for run 3 . The times are: upper left $t=16$, upper right $t=17$, lower left $t=18$ and lower right $\mathrm{t}=19$. 

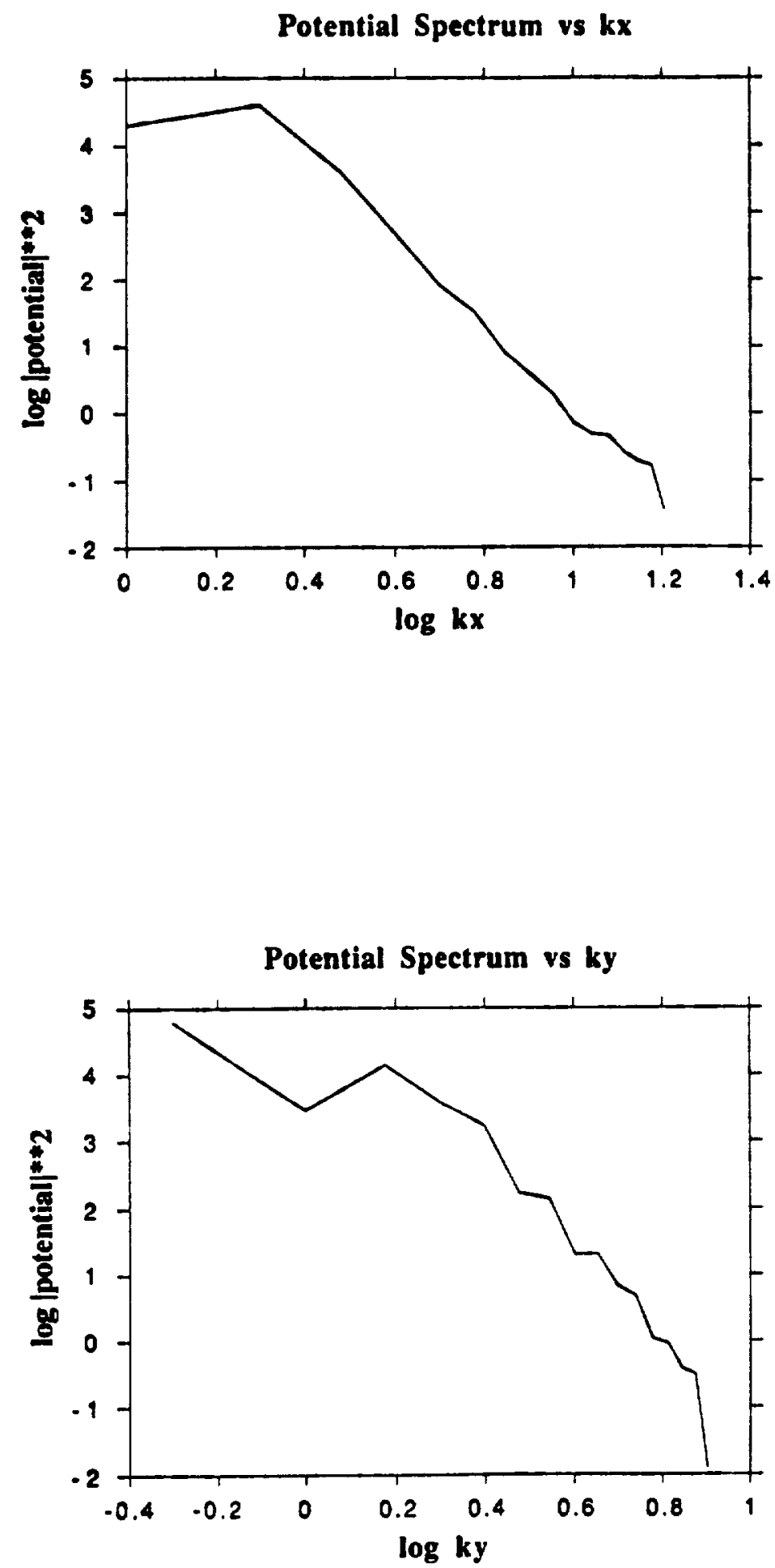

Fig 8 One dimensional potential power spectra for run 1 at time $t=20$. Upper panel is in the $x$-direction and lower panel is in the $y$-direction. 

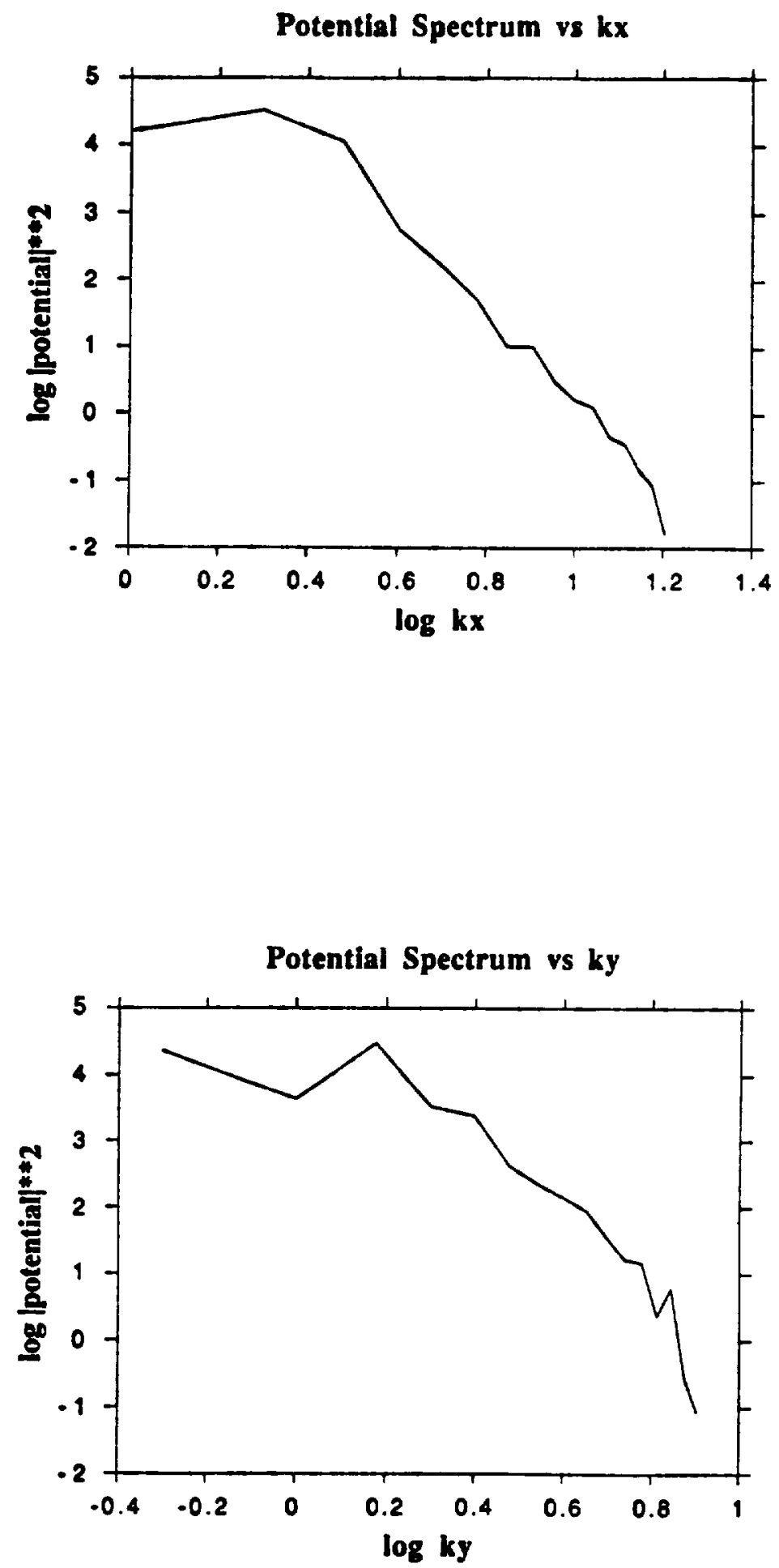

Fig 9 One dimensional potential power spectra for run 2 at time $t=20$. Upper panel is in the $x$-direction and lower panel is in the $y$-direction. 

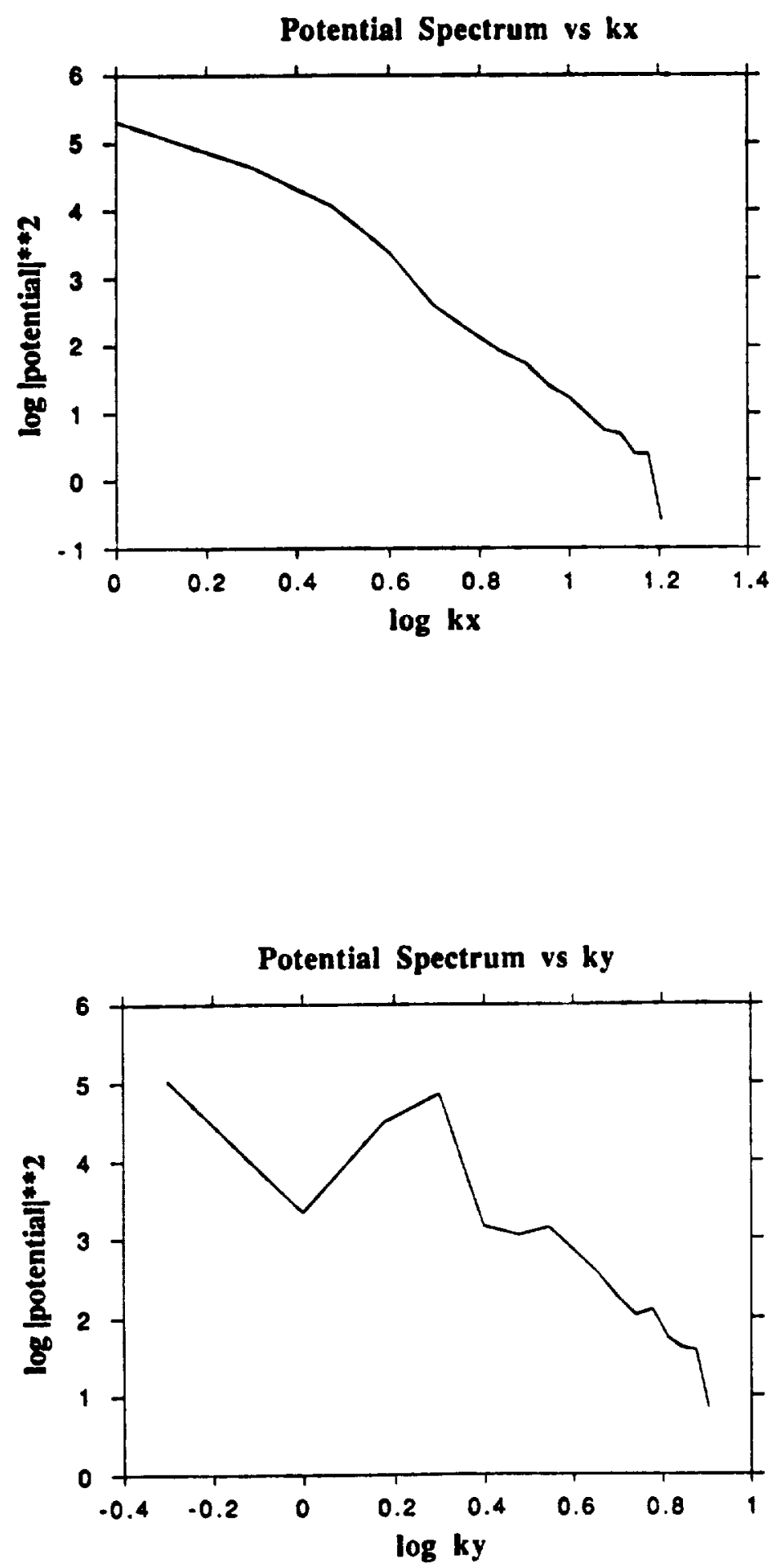

Fig 10 One dimensional potential power spectra for run 3 at time $t=16$. Upper panel is in the $x$-direction and lower panel is in the y-direction. 

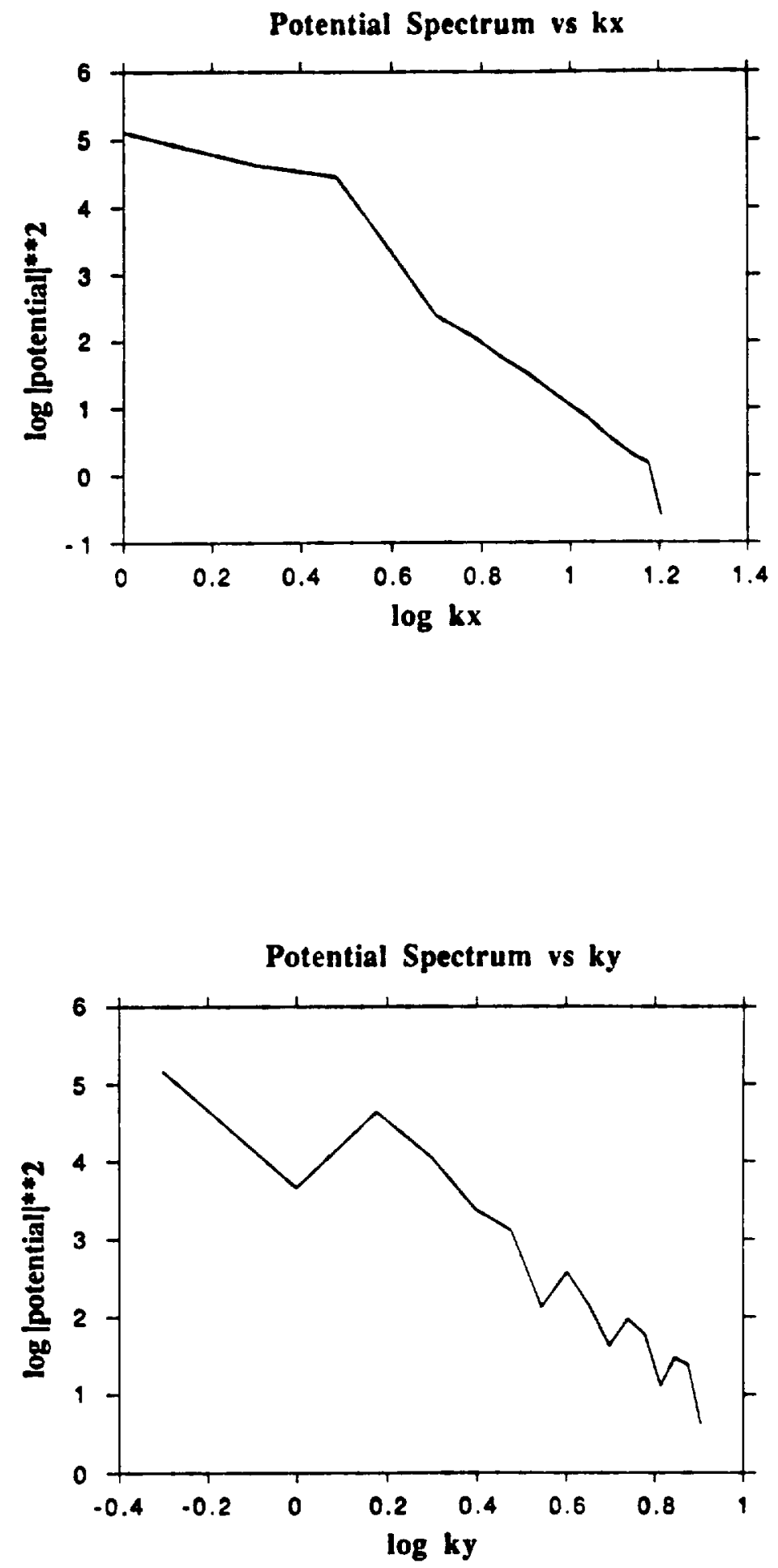

Fig 11 One dimensional potential power spectra for run 4 at time $\mathrm{t}=16$. Upper panel is in the $\mathrm{x}$-direction and lower panel is in the $y$-direction. 
